\title{
Surface Enhanced Raman Scattering for Quantification of p-Coumaric Acid Produced by Escherichia coli
}

Morelli, Lidia; Zor, Kinga; Jendresen, Christian Bille; Rindzevicius, Tomas; Schmidt, Michael Stenbæk; Nielsen, Alex Toftgaard; Boisen, Anja

Published in:

Analytical Chemistry

Link to article, DOI:

10.1021/acs.analchem.6b04428

Publication date:

2017

Document Version

Peer reviewed version

Link back to DTU Orbit

Citation $(A P A)$ :

Morelli, L., Zor, K., Jendresen, C. B., Rindzevicius, T., Schmidt, M. S., Nielsen, A. T., \& Boisen, A. (2017). Surface Enhanced Raman Scattering for Quantification of $p$-Coumaric Acid Produced by Escherichia coli. Analytical Chemistry, 89, 3981-3987. https://doi.org/10.1021/acs.analchem.6b04428

\section{General rights}

Copyright and moral rights for the publications made accessible in the public portal are retained by the authors and/or other copyright owners and it is a condition of accessing publications that users recognise and abide by the legal requirements associated with these rights.

- Users may download and print one copy of any publication from the public portal for the purpose of private study or research.

- You may not further distribute the material or use it for any profit-making activity or commercial gain

- You may freely distribute the URL identifying the publication in the public portal 


\title{
Surface enhanced Raman scattering for quantification of $p$-coumaric acid produced by Escherichia coli
}

\author{
Lidia Morelli $^{\mathrm{a} *}$, Kinga Zór ${ }^{\mathrm{a}}$, Christian Bille Jendresen ${ }^{\mathrm{b}}$, Tomas Rindzevicius ${ }^{\mathrm{a}}$, Michael Stenbæk \\ Schmidt $^{\mathrm{a}}$, Alex Toftgaard Nielsen ${ }^{\mathrm{b}}$, Anja Boisen ${ }^{\mathrm{a}}$ \\ ${ }^{a}$ Department of Micro- and Nanotechnology, Technical University of Denmark, 2800 Kgs. Lyngby, Denmark \\ ${ }^{\mathrm{b}}$ The Novo Nordisk Foundation Center for Biosustainability, Technical University of Denmark, 2800 Kgs. Lyngby, Den- \\ mark \\ * e-mail: 1mor@nanotech.dtu.dk; phone: +45 91734340.
}

\begin{abstract}
The number of newly developed genetic variants of microbial cell factories for production of biochemicals has been rapidly growing in recent years, leading to an increased need for new screening techniques. We developed a method based on surface-enhanced Raman spectroscopy (SERS) coupled with liquid-liquid extraction (LLE) for quantification of $p$-coumaric acid (pHCA) in the supernatant of genetically engineered Escherichia coli (E.coli) cultures. pHCA was measured in a dynamic range from $1 \mu \mathrm{M}$ up to $50 \mu \mathrm{M}$ on highly uniform SERS substrates based on leaning gold-capped nanopillars, which showed an in-wafer signal variation of only $11.7 \%$. LLE using dichloromethane as organic phase was combined with the detection in order to increase selectivity and sensitivity by decreasing the effect of interfering compounds from the analytes of interest. The difference in pHCA production yield between three genetically engineered $E$. coli strains was successfully evaluated using SERS and confirmed with high performance liquid chromatography. As this novel approach has potential to be automatized and parallelized, it can be considered for high throughput screening in metabolic engineering.
\end{abstract}

\section{INTRODUCTION}

Phenolic acids are molecules of high industrial and pharmaceutical importance. ${ }^{1}$ Due to their strong antioxidant and antimicrobial activity, ${ }^{2}$ they have several health benefits, and are used as natural additives in $\operatorname{cosmetics}^{3}$ or food products. ${ }^{4} p$ coumaric acid (pHCA) is widely available in seeds, fruit and vegetables, is a precursor of many phenolic compounds ${ }^{5}$ and is an excellent precursor for polymerization into high performance polymers. ${ }^{6}$ However, due to the lack of efficient extraction methods as well as limitations in its organic synthesis, there is a need for efficient and cost-effective methods for pHCA production. ${ }^{7}$ Metabolic engineering of microorganisms, such as Escherichia coli (E.coli) or Saccharomyces cerevisiae, has proven to be an effective approach for overproduction of $\mathrm{pHCA}$, through non-oxidative deamination of tyrosine (Tyr) by tyrosine-ammonia lyase (TAL). ${ }^{8-10}$ Different techniques, such as reversed-phase high performance liquid chromatography (RP-HPLC), ${ }^{8}$ thin layer chromatography ${ }^{11}$ and spectrophotometry ${ }^{10}$ are used for pHCA quantification in order to identify the best performing strain. ${ }^{12}$

These methods, although offering high accuracy and precision, can represent a major bottleneck in the screening of libraries of production organisms ${ }^{13}$ since they are often timeconsuming and require bulky and expensive instrumentation. Hence selective, fast, low-cost and high-throughput detection would represent an important innovation for small-molecule detection and quantification in this field.
Raman scattering based analysis of target molecules has many advantages, as it enables analysis of solutions with no or little sample pretreatment, while giving molecule-specific spectral information or vibrational "fingerprints". ${ }^{14}$ Since Raman scattering is a weak interaction, direct detection of trace amounts of molecules is challenging, which limits its applicability. ${ }^{15}$ The signal can be significantly enhanced utilizing the so-called surface-enhanced Raman scattering (SERS) technique, which is a well-established approach to obtain signals at low concentrations. ${ }^{16}$ Recently, SERS has grown into a powerful analytical tool due to its sensitivity, selectivity and speed of detection. ${ }^{17}$ It has been used for detection of nucleic acids and proteins, ${ }^{18}$ for rapid and selective detection of pathogens, ${ }^{19}$ biomarkers, ${ }^{20}$ hormones, ${ }^{21}$ additives ${ }^{22,23}$ and analytes from food samples. ${ }^{24}$ In order to effectively enhance the Raman signal, the analytes should be located within a few nanometers or preferably adsorbed onto the metal surface. ${ }^{25}$ Molecules containing sulphur or nitrogen atoms tend to interact strongly with gold, ${ }^{22}$ but species that bind via oxygen atoms show little affinity towards noble metal surfaces. ${ }^{26}$ Furthermore, detection of analytes in a complex media or in aqueous solutions with excess of salts is a challenge due to the presence of possible interfering compounds or formations of salt crystals. ${ }^{22,27}$ There are several approaches to increase the selectivity of detection in complex media either using surface functionalization, ${ }^{28}$ labeling techniques ${ }^{29}$ or sample pretreatment. ${ }^{30-33}$ 
pHCA detection in the complex bacterial supernatant from a TAL expressing microorganism has an additional challenge since Tyr is also present at various quantities at the end of the reaction, depending on the pHCA production efficiency of the specific strain. Given the similarities in their molecular structure ${ }^{34} \mathrm{pHCA}$ and Tyr spectra overlap with similar vibrational features, which makes identification and quantification more challenging. Therefore, a sample pre-treatment method needs to be implemented to separate the compounds. Liquid-liquid extraction (LLE) is a common method for extraction of phenolic compounds from fruit, vegetables, ${ }^{35}$ food samples ${ }^{36}$ and from bacterial supernatant. ${ }^{37} \mathrm{~A}$ wide variety of protocols have been developed in the last few decades, based on different combinations of solvents as organic phase for sample extraction and/or up concentration. ${ }^{38,39}$ A major advantage of LLE in the case of pHCA detection is that the apolar pHCA will be extracted to the organic phase whereas polar molecules (e.g Tyr) will remain in the aqueous phase. Moreover using the organic phase for SERS-based detection results in an improved leaning of metal-capped nanopillars, leading to increased enhancement of the Raman signal. ${ }^{33}$

In this paper, we describe the development of a SERS-based method for quantification of pHCA when screening genetically modified $E$. coli strains. To the best of our knowledge, this work is the first reported example of a SERS-based quantification for screening of genetically modified organisms. The SERS substrates used in this work were fabricated using a fast and cost-effective method developed by Schmidt et al., ${ }^{40-42}$ which yields uniform and stable SERS-active surfaces. The goal of this work was to develop a SERS-based detection method combined with a simple extraction procedure such as LLE, which can be used for quantification of metabolites (e.g. pHCA).

\section{EXPERIMENTAL SECTION}

Chemicals. $p$-coumaric acid (pHCA), tyrosine (Tyr), ethanol (EtOH), dichloromethane (DCM), diethyl ether (DE), ethyl acetate (EA), octanol, sodium hydroxide $(\mathrm{NaOH})$, hydrochloric acid $(\mathrm{HCl}), \mathrm{HK}_{2} \mathrm{PO}_{4}$ and $\mathrm{H}_{2} \mathrm{KPO}_{4}$ were used for sample preparation for HPLC and SERS experiments. For pHCA production assays the $E$. coli were grown in minimal M9 medium, ${ }^{43}$ containing $12.8 \mathrm{~g} / \mathrm{L} \mathrm{Na}_{2} \mathrm{HPO}_{4} \cdot 7 \mathrm{H}_{2} \mathrm{O}, 3.0 \mathrm{~g} / \mathrm{L} \mathrm{KH}_{2} \mathrm{PO}_{4}$, $0.5 \mathrm{~g} / \mathrm{L} \mathrm{NaCl}, 1.0 \mathrm{~g} / \mathrm{L} \mathrm{NH}_{4} \mathrm{Cl}, 2 \mathrm{mM} \mathrm{MgSO}$, $0.1 \mathrm{mM} \mathrm{CaCl}_{2}$, and 1:2000 volume of trace elements (prepared as follows: 10 $\mathrm{g} / \mathrm{L} \mathrm{FeCl}_{3} \cdot 6 \mathrm{H}_{2} \mathrm{O}, 2 \mathrm{~g} / \mathrm{L} \mathrm{ZnSO} \mathrm{Zn}_{4} \cdot 7 \mathrm{H}_{2} \mathrm{O}, 0.4 \mathrm{~g} / \mathrm{L} \mathrm{CuCl} \mathrm{Cu}_{2} \cdot 2 \mathrm{H}_{2} \mathrm{O}, 1$ $\mathrm{g} / \mathrm{L} \mathrm{MnSO}_{4} \cdot \mathrm{H}_{2} \mathrm{O}, 0.6 \mathrm{~g} / \mathrm{L} \mathrm{CoCl}_{2} \cdot 6 \mathrm{H}_{2} \mathrm{O}$ and $3.2 \mathrm{~mL} / \mathrm{L}$ of $0.5 \mathrm{M}$ EDTA pH8 mixed and autoclaved ${ }^{44}$ ), $10 \mathrm{~g} / \mathrm{L}$ glucose, $2 \mathrm{mM}$ tyrosine, $1 \mathrm{mM}$ IPTG, as well as Wolfe's Vitamin solution (final concentrations of $10 \mu \mathrm{g} / \mathrm{L}$ pyridoxine- $\mathrm{HCl}, 5 \mu \mathrm{g} / \mathrm{L}$ thiamine- $\mathrm{HCl}, 5 \mu \mathrm{g} / \mathrm{L}$ riboflavin, $5 \mu \mathrm{g} / \mathrm{L}$ nicotinic acid, $5 \mu \mathrm{g} / \mathrm{L}$ calcium D-(+)-pantothenate, $5 \mu \mathrm{g} / \mathrm{L}$ p-aminobenzoic acid, 2 $\mu \mathrm{g} / \mathrm{L}$ biotin, $2 \mu \mathrm{g} / \mathrm{L}$ folic acid, $5 \mu \mathrm{g} / \mathrm{L}$ lipoic acid, $0.1 \mu \mathrm{g} / \mathrm{L}$ vitamin B12) purchased from ATCC® (LGC Standards, UK). Furthermore, plasmids were maintained by selection using 34 $\mathrm{mg} / \mathrm{L}$ chloramphenicol and $50 \mathrm{mg} / \mathrm{L}$ spectinomycin as antibiotics added to the medium. Aqueous solutions were prepared in ultrapure water obtained from a Milli-Q ${ }^{\circledR}$ water purification system (Millipore Corporation, Billerica, MA, USA). All the chemicals were purchased from Sigma-Aldrich ${ }^{\circledR}$ Co. (St. Louis, MO, USA) unless otherwise stated.
E. coli culture. E. coli strains CBJ786, CBJ892 and CBJ800 were derived as described by Jendresen et al. ${ }^{12}$ from the expression strain BL21(DE3)pLysS (Invitrogen/Life Technologies), carrying the extrachromosomal plasmid pCDFDuet-1 (CBJ786) and derivative plasmids encoding the tyrosine ammonia-lyases R XAL (CBJ792) and FjTAL (CBJ800). The strains were grown in $180 \mathrm{~mL}$ M9 medium in $500 \mathrm{~mL}$-flasks in a temperature-controlled orbital shaker $(30$ $\left.{ }^{\circ} \mathrm{C}, 250 \mathrm{rpm}\right) .30 \mathrm{~mL}$ samples were withdrawn at $24 \mathrm{~h}$, and supernatant was obtained by centrifugation (10 $\mathrm{min}$ at 10000 $g, 4{ }^{\circ} \mathrm{C}$ ) and filtration through a $0.2 \mu \mathrm{m}$ filter.

Sample preparation and extraction. pHCA stock solutions $(100 \mathrm{mM})$ were prepared in $99 \% \mathrm{EtOH}$ and diluted at different concentrations in DCM for the SERS calibration standards, or in M9 medium for optimization of the extraction process and the HPLC calibration standards. Similarly, Tyr was dissolved in a solution of $\mathrm{NaOH} 0.1 \mathrm{mM}$ in ultrapure water at $\mathrm{pH} 10$ to prepare a $10 \mathrm{mM}$ stock, and diluted in $\mathrm{M} 9$ medium. The sample preparation including the extraction of pHCA based on Krygier et al. ${ }^{45}$ is presented in Figure 1.

$500 \mu \mathrm{L}$ of bacterial supernatant or M9 medium containing pHCA and/or Tyr were acidified with $50 \mu \mathrm{L} \mathrm{32 \%} \mathrm{HCl}(\mathrm{aq})$ and vortexed for optimal mixing. After the acidification step, 500 $\mu \mathrm{L}$ DCM was added to the sample and vortexed again, followed by static incubation for $30 \mathrm{~min}$ at room temperature.
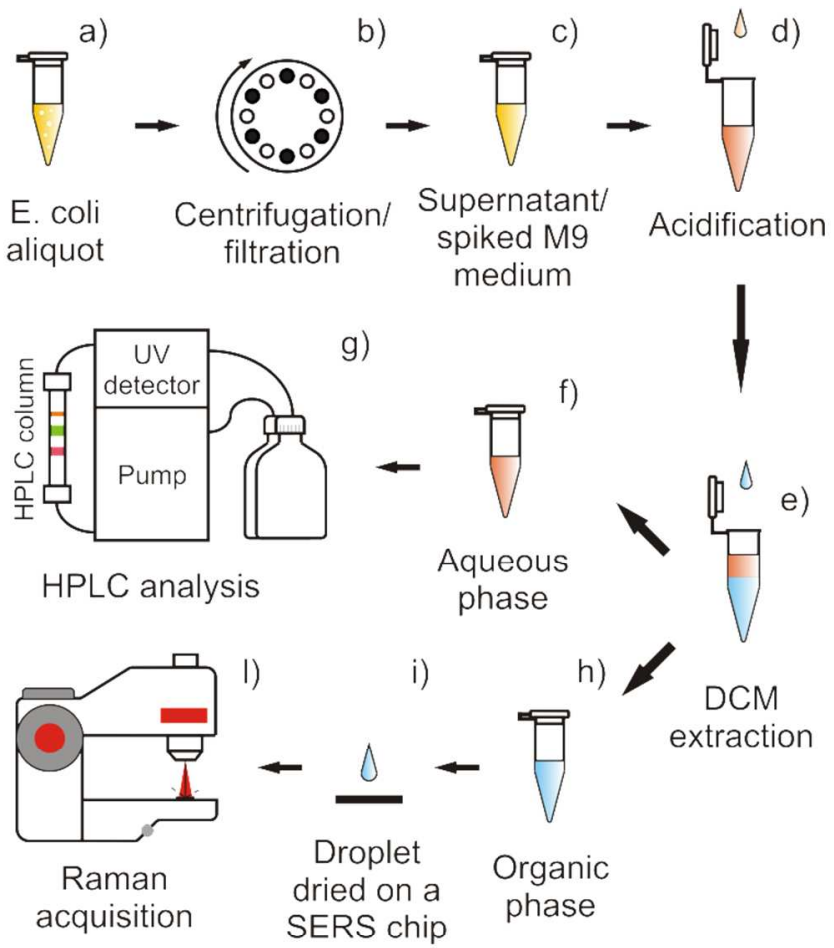

Figure 1. Procedure for the extraction of pHCA from E.coli supernatant or spiked M9 medium. The E.coli aliquot (a) was centrifuged and filtered (b) in order to extract supernatant (c). The sample (supernatant or spiked M9 medium) was then acidified (d) and DCM was added (e). The amount of pHCA in the aqueous phase after extraction (f) was quantified by HPLC (g), whereas a droplet of the organic phase was dried on a SERS chip (h, i) and quantified through Raman acquisition (l). 
The aqueous phase was removed and diluted 10 times with phosphate buffer $(\mathrm{pH} 8,0.04 \mathrm{M})$ in order to increase the $\mathrm{pH}$ to 6 for HPLC analysis, whereas the organic phase was used for SERS analysis.

HPLC analysis. Calibration standards in M9 medium ranging from $1 \mu \mathrm{M}$ to $10 \mathrm{mM}$ were diluted 10 times in phosphate buffer, $\mathrm{pH}$ 8. Injections $(5 \mu \mathrm{L})$ were separated on a Discovery HS F5 column ( $3 \mu \mathrm{m}$ particle size, $15 \mathrm{~cm} \mathrm{x} 4.6 \mathrm{~mm}$ ) at $30^{\circ} \mathrm{C}$ in an HPLC system (Thermo Scientific, Waltham, MA USA 02451 ) as previously described. ${ }^{12}$ Elution was performed using a gradient with two solvents: $10 \mathrm{mM}$ ammonium formate adjusted to $\mathrm{pH} 3.0$ with formic acid (A) and acetonitrile (B) running at $0.7 \mathrm{~mL} / \mathrm{min}$, starting at $5 \% \mathrm{~B}$. The fraction of $\mathrm{B}$ increased linearly from $5 \%$ to $60 \%$ from $1.5 \mathrm{~min}$ to $7 \mathrm{~min}$ after injection. Then the fraction of B decreased back to $5 \%$ between 9.5 and $9.6 \mathrm{~min}$, and remained there until $12 \mathrm{~min}$. pHCA was detected by absorbance at $333 \mathrm{~nm}$.

Preparation of gold-capped nanopillars (AuNP). The SERS substrates were fabricated on $10 \mathrm{~cm}$ diameter polished silicon wafer with a maskless fabrication process. ${ }^{46}$ The fabrication process was followed as described by $\mathrm{Wu}$ et al., ${ }^{47}$ using 4 min etching time, 1 min $\mathrm{O}_{2}$ plasma treatment and depositing $220 \mathrm{~nm}$ of $\mathrm{Au}$ on the etched wafers at a rate of $10 \AA / \mathrm{s}$ instead of $\mathrm{Cr}$ and $\mathrm{Ag}$. The resulting structures were $900 \mathrm{~nm}$ in height, $130 \mathrm{~nm}$ in width and with a density of about 23 pillars $/ \mu \mathrm{m}^{2}$. The wafers were diced into $4 \times 4 \mathrm{~mm}$ chips using a Laser Micromachining tool (3D-Micromac AG, D-09126 Chemnitz, Germany), and stored under vacuum before use.

SERS measurements. All SERS measurements were recorded with a DXR Raman Microscope (Thermo Fisher Scientific, Inc.,Waltham, MA, USA.). The optical microscope is coupled to a single grating spectrometer with $5 \mathrm{~cm}^{-1}$ FWHM spectral resolution and $\pm 2 \mathrm{~cm}^{-1}$ wavenumber accuracy. All SERS spectra were collected at $780 \mathrm{~nm}$ with a laser power of 1 $\mathrm{mW}$, using a 10x objective lens, a $25 \mu \mathrm{m}$ slit and an estimated laser spot of $3.6 \mu \mathrm{m}$ diameter. The spectrum was acquired 3 times for $3 \mathrm{~s}$ in each spot. Droplets of DCM solutions $(5 \mu \mathrm{L})$ were deposited on each SERS chip and dried before acquisition. The droplets wetted the surface of the chip completely, and dried in a few seconds. The acquisition was performed over a $2.5 \times 2.5 \mathrm{~mm}^{2}$ area in the center of the chip, with a 25 points map with $500 \mu \mathrm{m}$ signal collection step with an overall acquisition time of $5 \mathrm{~min}$. In case of aqueous samples, smaller droplets $(1 \mu \mathrm{L})$ were used to reduce the amount of salts on the sensing surface, and acquisitions were performed with smaller maps in salt-free spots. In each experiment, all the SERS chips were taken from the same wafer, and a calibration curve was acquired each time for quantification. Unless stated otherwise, triplicates were acquired for each sample.

Spectral data analysis. All the spectral data were collected in the region $200-3400 \mathrm{~cm}^{-1}$. Prominent peaks were observed at 1169 and $1250 \mathrm{~cm}^{-1}$, which correspond to $\mathrm{CH}$ bending and CO stretching motions, and 1607 and $1629 \mathrm{~cm}^{-1}$, which are related to aromatic ring stretch motion. ${ }^{48}$ The data processing included background correction with the method described by Mazet at al. ${ }^{49}$ After averaging and removing the outliers, the height of the characteristic peak at $1169 \mathrm{~cm}^{-1}$ was determined for each map with a linear background correction in the peak region. For calibration curves, the peak height was plotted against the corresponding concentration, and the Origin Pro
(OriginLab, Northampton, MA, US) linear fitting tool was used to obtain the equation. The reproducibility of the signal was evaluated as the standard deviation of the average peak heights extracted from each chip.

\section{RESULTS AND DISCUSSION}

Optimization of pHCA detection. In this paragraph we explain the advantages of performing LLE before SERS-based sensing. We carried out experiments to find the most suitable organic solvent for LLE and SERS-based detection of pHCA. In order to evaluate the possibility of pHCA detection directly from bacterial supernatant we carried out experiments both in aqueous solution and organic solvent. The effectiveness of solvent extraction against Tyr was also assessed.

The modified strains expressing TAL were cultured in a relatively complex mixture, (M9 medium, as described in Experimental section) with appropriate salts, antibiotics, nutrients, and Tyr at starting concentration up to $2 \mathrm{mM}$, according to the methods described by Jendresen et al. ${ }^{8}$ As previously reported, ${ }^{27}$ supernatant samples as well as spiked M9 medium need to be diluted at least 10 times with ultrapure water in order to be able to detect the SERS signal of pHCA, due to salt clogging of the active surface (Figure 3 (b) and (c)). The efficiency of TAL enzymatic reaction (Figure 2 (a)) is dependent on the genetic modification of the bacteria, therefore a certain amount of Tyr can be found as well in the supernatant at different time points during the culture (Table S1). The structure of Tyr is similar to pHCA, apart from an amine group, which preferentially interacts with gold. ${ }^{50}$ Hence, even though the Raman activity of Tyr is weaker than pHCA, the SERS signal
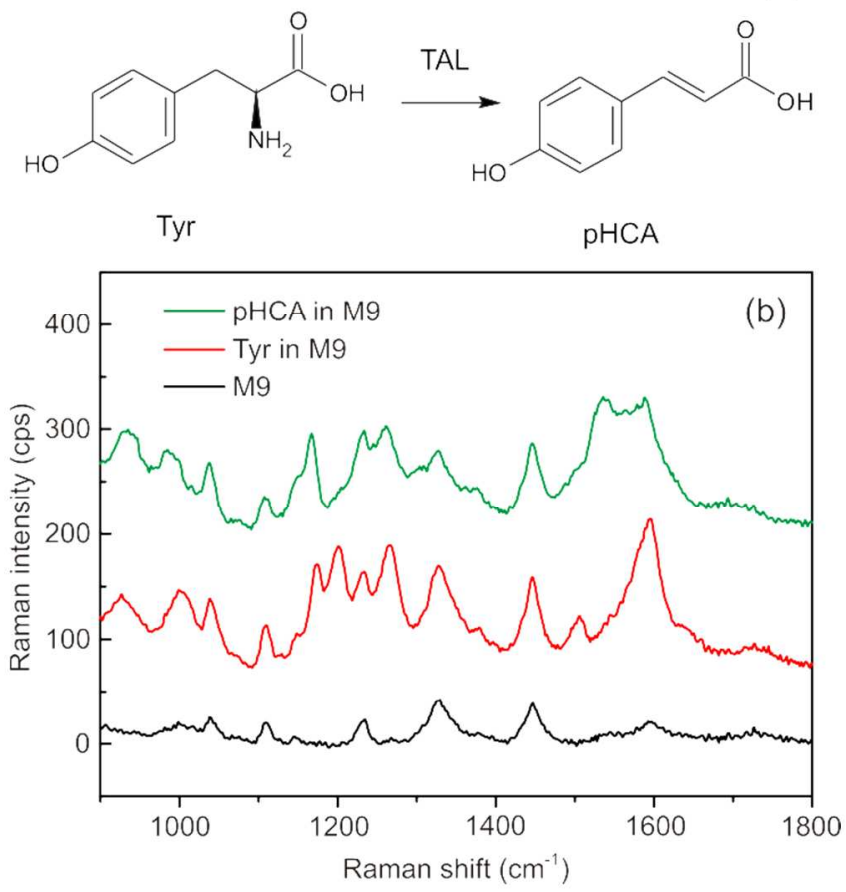

Figure 2. (a) TAL enzymatic reaction. (b) SERS spectra of $50 \mu \mathrm{M}$ pHCA and Tyr spiked M9 medium subsequently diluted 10-fold with ultrapure water. The graphs were shifted with arbitrary offsets for ease of representation. 
is comparable on AuNP substrates in the fingerprint region (Figure 2 (b)) when measuring pHCA and Tyr spiked in M9 media. As their spectral features overlap, when both molecules are in solution it is difficult to separate each contribution at the wavelength of the characteristic pHCA peak. Furthermore, as previously reported, ${ }^{33}$ SERS substrates based on hydrophobic leaning nanostructures give a better signal when wetted with organic solvents. Using organic solvents improves the diffusion of analytes in between and under the gold nanoparticles, and increases the lateral capillary force between adjacent nanopillars, creating more consistent leaning and higher enhancement. Hence, bacterial supernatant, as an aqueous solution, does not represent the ideal working condition for AuNP. ${ }^{27}$ Therefore, there is a need for sample pretreatment in order to address these issues.

We implemented a sample pretreatment step prior to SERS analysis based on LLE as presented in Figure 1. To find the
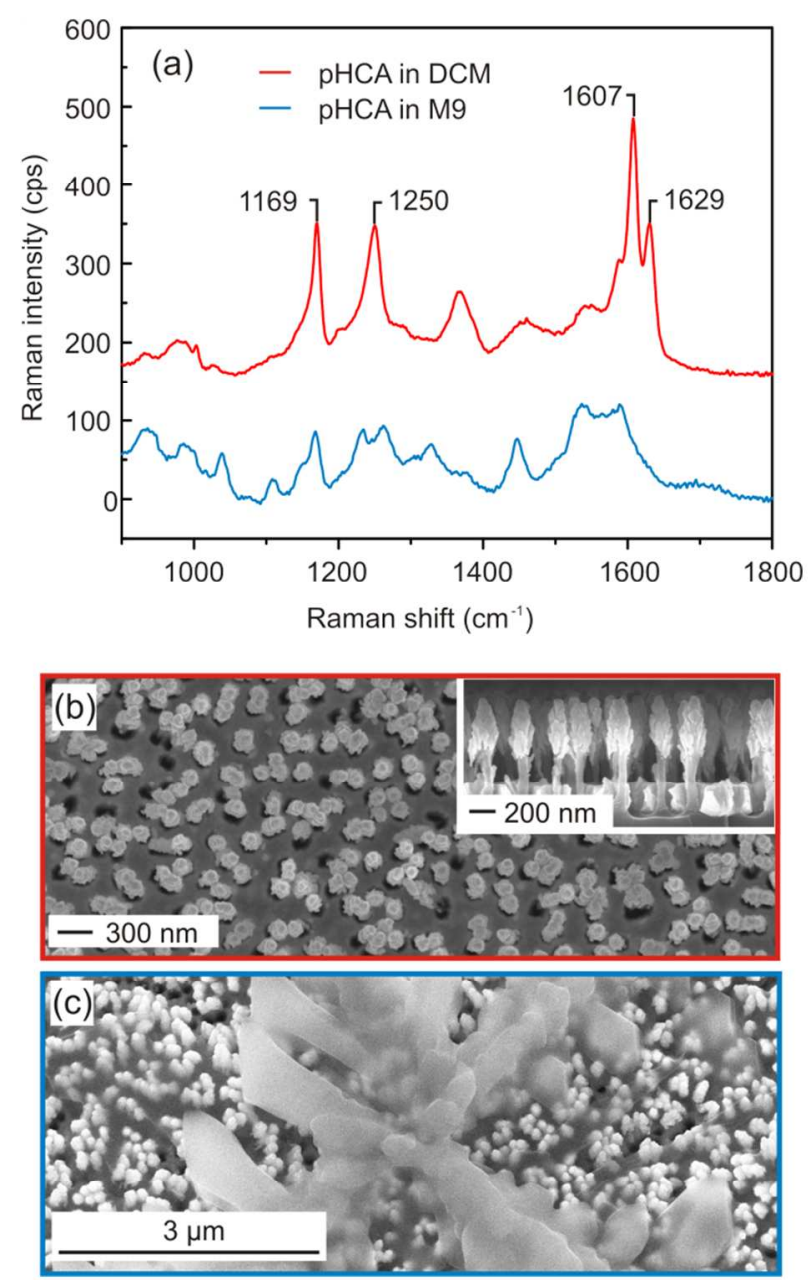

Figure 3. (a) SERS spectra of $1 \mu \mathrm{L}$ of $50 \mu \mathrm{M}$ pHCA in DCM (red) and in M9 medium 10 times diluted with ultrapure water (blue). The red graph was shifted with an arbitrary offset for ease of representation (see Spectral data analysis for band assignment). (b) Clean surface of a SERS chip exposed to pHCA in DCM, with a side view of the AuNP (inset). (c) Salts crystallized on a SERS substrate after exposure to diluted M9 medium.

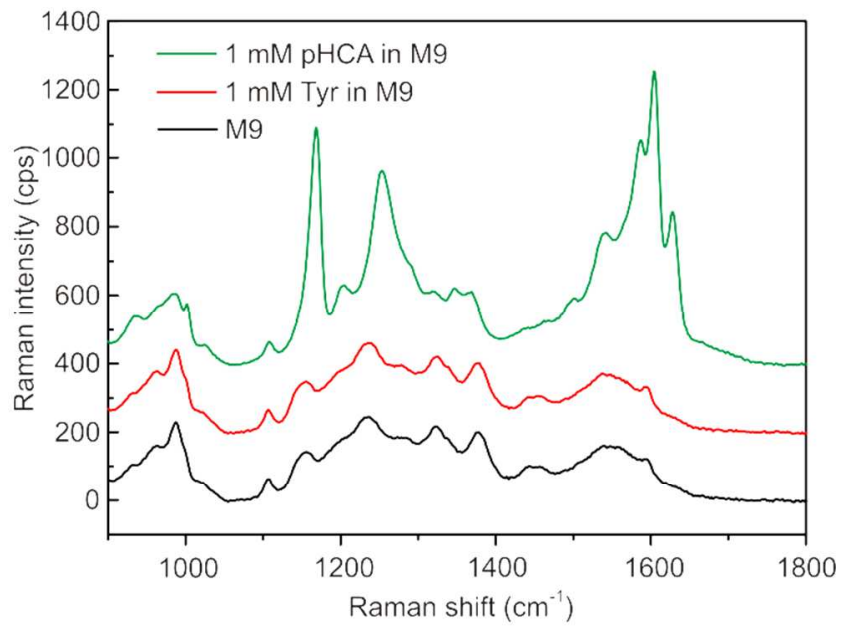

Figure 4. LLE extraction of $1 \mathrm{mM}$ pHCA spiked in M9 medium (green), $1 \mathrm{mM}$ Tyr (red) and M9 medium without pHCA or Tyr (black). The graphs were shifted with arbitrary offsets for ease of representation.

most suitable solvent for LLE we evaluated DCM as well as EA, DE and octanol, ${ }^{51,52,37}$ non-water-miscible organic solvents which are commonly used for extraction of phenolic compounds. Solvent compatibility with SERS-based sensing, background signal and peak interference with pHCA after M9 and supernatant extraction were evaluated. Octanol was excluded due to slow evaporation. When using EA in the LLE process the pHCA signal was 12 times lower than in the case of DE and DCM (Figure S1). DCM was found suitable for LLE combined with SERS detection, since when using DE the control sample showed significant background signal at 1169 $\mathrm{cm}^{-1}$ which was not observed in case of DCM (Figure S2).

Additionally, DCM was chosen as organic solvent based on its Raman signal ${ }^{53}$ after comparison with $\mathrm{DE}^{51}$ and EA. ${ }^{52} \mathrm{In}-$ deed, a droplet of DCM spiked with pHCA showed higher signal compared to 10 times diluted M9 medium spiked with the same concentration (Figure 3 (a)).

LLE proved effective for removal of Tyr. At low $\mathrm{pH}$, $\mathrm{pHCA}$ is neutral $\left(\mathrm{pK}_{\mathrm{na}} \sim 4.9\right)^{54}$, whereas the amine group of Tyr is charged. Hence, the acidification step increases pHCA affinity towards the organic phase, whereas Tyr stays in the aqueous phase (Figure 4). Besides Tyr, other charged molecules and salts also have affinity for the aqueous phase so they do not interfere with SERS measurements.

pHCA quantification. Calibration curves were constructed using different concentrations from $1 \mu \mathrm{M}$ to $175 \mu \mathrm{M}$ of pHCA in DCM. Figure 5 shows a typical calibration curve obtained using different $\mathrm{pHCA}$ concentrations in DCM on SERS substrates. The amplitude of the representative peak at $1169 \mathrm{~cm}^{-1}$ was plotted as a function of pHCA concentration. The substrates show a linear response from $1 \mu \mathrm{M}$ up to $50 \mu \mathrm{M}$ (Figure 5 , inset), which was used as calibration curve for quantification of unknown samples. 


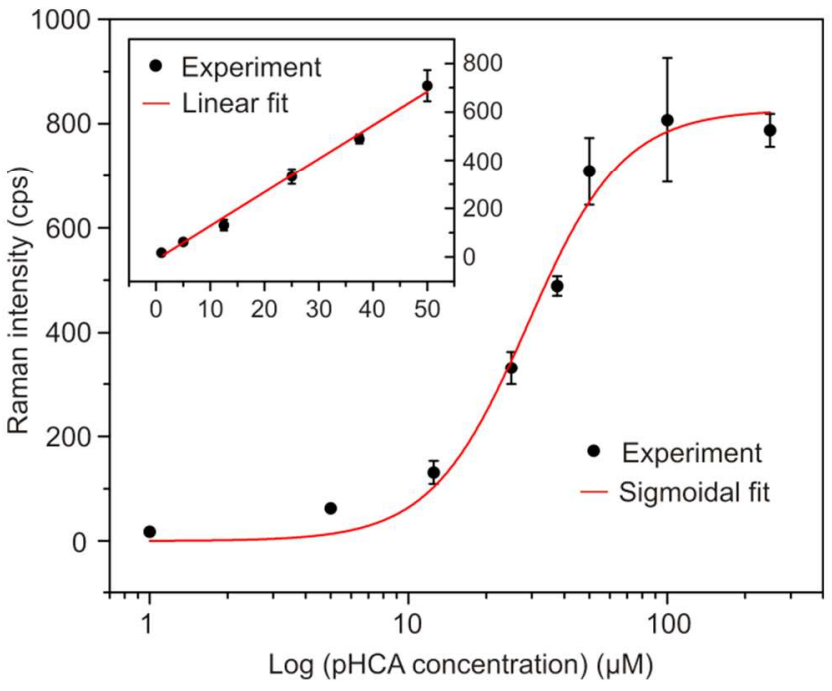

Figure 5. Calibration curve of pHCA, with SERS intensity at $1169 \mathrm{~cm}^{-1}$ as a function of pHCA concentration. Each point represents the average of 3 maps, with error bars representing standard deviation. The experimental data were fitted with Hill's equation (sigmoidal curve). The linear part of the calibration curve is presented in the inset.

The reproducibility of SERS substrates was investigated inwafer, in-batch and batch-to-batch. Two batches of three wafers were fabricated for this purpose, analyzing the intensity of pHCA peak at $1169 \mathrm{~cm}^{-1}$ in a range of concentrations between 10 and $100 \mu \mathrm{M}$. A 25 points map was acquired from each chip, and the reproducibility was evaluated as the relative standard deviation (RSD) over the average values extracted from each map. In-wafer RSD was found to be $11.7 \%$ (5 concentration points, each measured in triplicates, $n=15)$. The inbatch variation was $21.7 \%$ ( 3 concentrations points on 3 wafers, each measured in duplicates, $\mathrm{n}=18$ ) and the batch-tobatch variation was found to be $48.7 \%$ ( 3 concentration points on 6 wafers, each measured in duplicates, $\mathrm{n}=36$ ). The inbatch and batch-to-batch variations were overcome by performing calibrations for each wafer, given the good in-wafer reproducibility.

Validation of LLE with HPLC. In order to evaluate the effect of the incubation time on the extraction efficiency, 1 $\mathrm{mM}$ pHCA in $\mathrm{M} 9$ medium was extracted following the protocol described in Experimental section using different extraction times $(5,15,30$ and $60 \mathrm{~min})$ (Figure S3). The extraction time was defined as the time the sample was in contact with DCM, and the extraction efficiency as the percentage of pHCA found in the organic phase compared to the starting concentration. While $5 \mathrm{~min}$ of incubation proved sufficient (Figure S3), 30 min was chosen as an optimal incubation time to ensure good phase separation in the vials before removing the organic phase.

The extraction efficiency was evaluated using different concentrations of pHCA spiked in M9 medium $(100,175,250$, $500 \mu \mathrm{M})$, analyzed with both HPLC and SERS. The HPLC measurements carried out on the aqueous phase showed an average extraction efficiency of $9.8 \pm 1.1 \%$, constant over different starting concentrations. Hence, the amount of ex- tracted $\mathrm{pHCA}$ was linear with the starting concentration. Furthermore, pHCA concentration extracted to the organic phase was quantified with SERS and plotted versus the starting concentration values (Figure 6). We observed a close correlation between pHCA concentration obtained with SERS and HPLC. This demonstrates that SERS can be effectively used for quantification of pHCA in M9 medium, in combination with LLE.

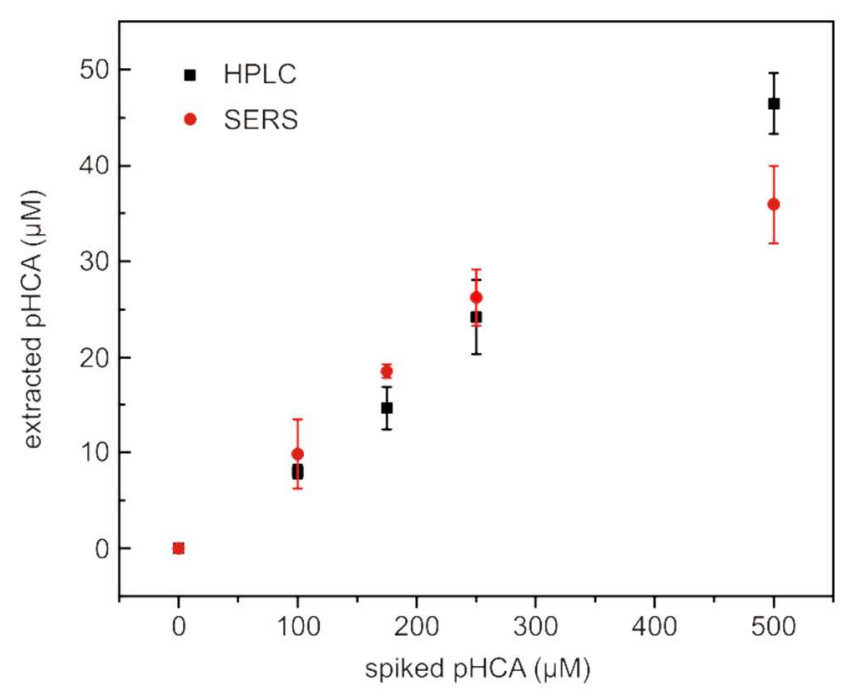

Figure 6. Extracted amount of pHCA measured with both HPLC and SERS. Each HPLC point in the graph is the average of independent triplicates, with error bars representing standard deviation. Each SERS point represents independent triplicates, each measured in duplicates, with error bars representing the overall standard deviation.

Quantification of pHCA from bacterial supernatant. In order to test the applicability of the method for screening of genetically modified organisms, the LLE process was carried out on supernatants of bacterial cultures. A control, nonpHCA-producing strain (CBJ786) and two pHCA-producing strains (CBJ792 and CBJ800) were grown in M9 minimal medium. The two pHCA producing strains expressed different TAL enzymes thereby resulting in different synthesis yields of pHCA as previously described. ${ }^{12}$ Samples were taken after 24 $\mathrm{h}$ of culturing. The extracts were measured with SERS (Figure 7 (a)) and HPLC following the steps presented in Figure 1. Based on the HPLC data the LLE extraction efficiency from supernatant was comparable $(10.5 \pm 1.1 \%)$ with the data obtained from pHCA spiked M9 medium. The extracts were measured with SERS in different dilutions as presented in Figure 7 (b). When measuring the undiluted extracts, the Raman intensity was lower than expected based on HPLC results. The low signal could be due to interfering compounds from the sample matrix, which do not have Raman activity, but were extracted to the organic phase with pHCA. These interfering compounds cover part of the sensing area, leading to surface fouling and decrease in SERS intensity. Matrix dilu$\operatorname{tion}^{55}$ is commonly used to dilute out the interfering compounds if the analyte concentration is high enough to be detected even after being diluted. In order to obtain an accurate 

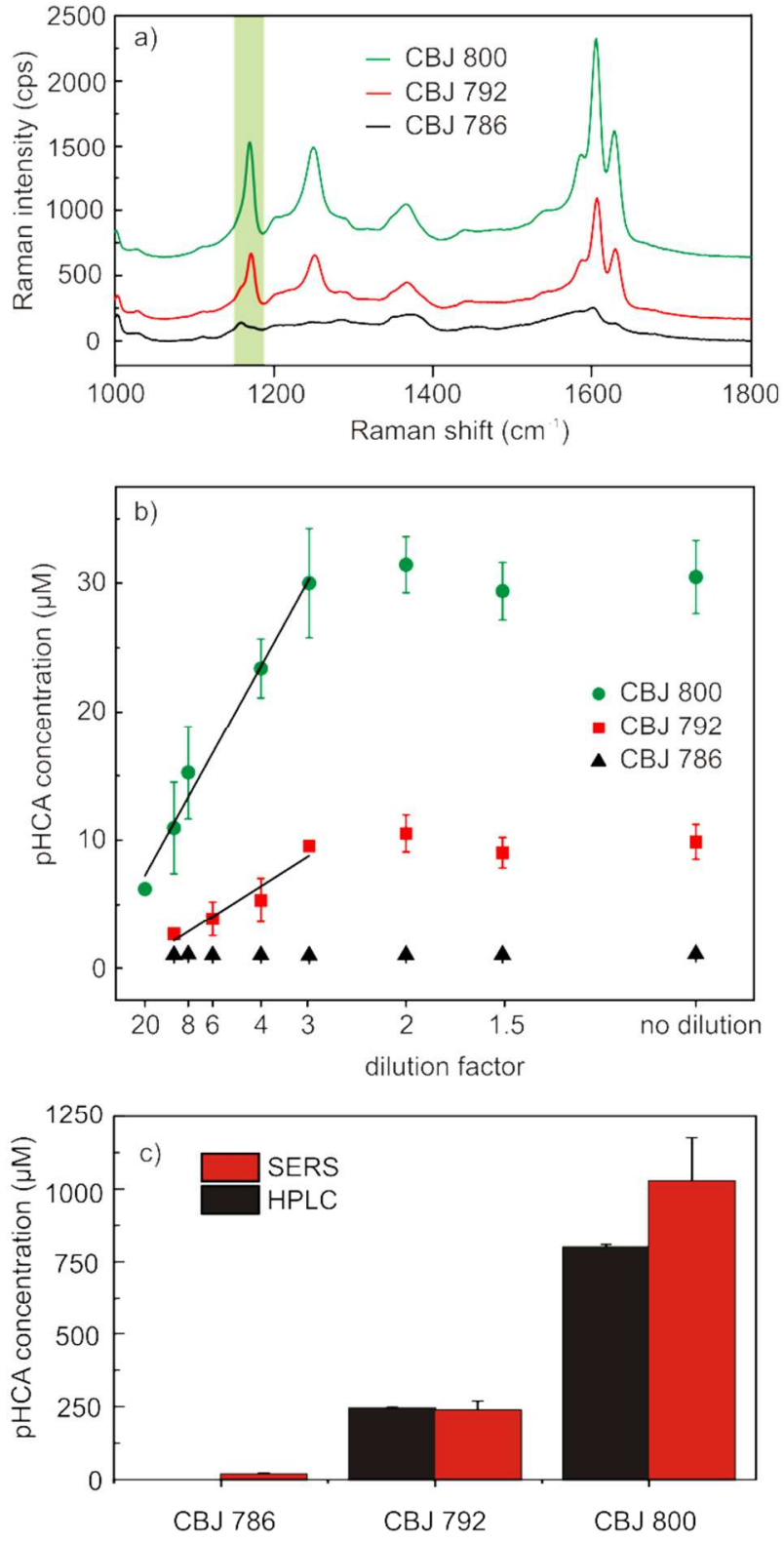

Figure 7. (a) SERS spectra from supernatant extracts after DCM extraction. The peak region is highlighted in light green, and the graphs were shifted with arbitrary offsets for ease of representation. (b) SERS intensity at $1169 \mathrm{~cm}^{-1}$ versus dilution of sample extracts. The graph is the combination of datasets obtained with 3 independent experiments, each measured in duplicates. The error bars represent the overall standard deviation. (c) Comparison between pHCA produced by E. coli strains calculated with SERS and HPLC.

quantification of the extracted $\mathrm{pHCA}$, the extracts were therefore diluted at different dilution factors $(1.5,2,3,4,6,8,10$ and 20) in DCM and measured. As it can be observed in Figure 7 (b) the Raman intensity decreased with the sample dilution except for the control strain. The data points in the linear part of the curves, with dilution factors higher than 3 , were used for quantification. The concentration of pHCA for CBJ800 and CBJ792 was found to be $1026.9 \pm 149.6 \mu \mathrm{M}$ and $237.2 \pm 31.5 \mu \mathrm{M}$ respectively, with results comparable to
HPLC $(800.3 \pm 10.1 \mu \mathrm{M}$ and $243 \pm 5.3 \mu \mathrm{M})$, as shown in Figure 7 (c).

\section{CONCLUSIONS}

In this work we show that SERS can be effectively used as an alternative to standard analytical methods for differentiation of $E$. coli producing different amounts of pHCA. The SERS substrates provided stable and reproducible signal within each wafer, and the combination with LLE successfully overcame the limitations related to SERS-based sensing in a complex medium.

The data obtained from HPLC and SERS analysis were closely correlated for both spiked medium and supernatant samples, validating the results obtained with SERS. In the developed method the detection time and the amount of organic solvent per sample were lower than for the HPLC assay, with comparable detection limit.

The presented work shows the potential and possibilities offered by SERS-based detection combined with LLE for prescreening of genetically modified microorganisms. Furthermore, the potential of the proposed method could be fully developed through integration in an automated and highthroughput microfluidic platform, leading to fast and costefficient routine analysis.

\section{ASSOCIATED CONTENT}

\section{Supporting Information}

Additional material about Tyr and pHCA content in E. coli supernatant during bacterial culture and experiments with different extraction solvents.

The Supporting Information is available free of charge on the ACS Publications website.

\section{AUTHOR INFORMATION}

\section{Corresponding Author}

\section{Author Contributions}

All authors have given approval to the final version of the manuscript.

\section{ACKNOWLEDGMENT}

Tommy Sonne Alstrøm and Prof. Henrik Flyvbjerg for help with data treatment, Pep Charusanti for troubleshooting with HPLC, Kaiyu $\mathrm{Wu}$ for discussion about SERS substrates.

The research is funded by the Danish National Research Foundation (DNRF122), Villum Fonden (Grant No. 9301), the European Research Council (320535, 'HERMES'). Christian Bille Jendresen was supported by The Novo Nordisk Foundation (Grant No. NNF15OC0015246).

\section{REFERENCES}

(1) Encyclopaedia of Occupational Health and Safety: Guides indexes, directory; Mager Stellman, J., Ed.; Geneva, International Labour Office, 1998.

(2) Kadoma, Y.; Fujisawa, S. Molecules 2008, 13, 2488-2499.

(3) Ribeiro, A.; Estanqueiro, M.; Oliveira, M.; Sousa Lobo, J. Cosmetics 2015, 2, 48-65.

(4) Balasundram, N.; Sundram, K.; Samman, S. Food Chem. 2006, 99, 191-203. 
(5) Nishiyama, Y.; Yun, C.-S.; Matsuda, F.; Sasaki, T.; Saito, K.; Tozawa, Y. Planta 2010, 232, 209-218.

(6) Thi, T. H.; Matsusaki, M.; Shi, D.; Kaneko, T.; Akashi, M. J. Biomater. Sci. Polym. Ed. 2008, 19, 75-85.

(7) Santos, C. N. S.; Koffas, M.; Stephanopoulos, G. Metab. Eng. 2011, 13, 392-400.

(8) Jendresen, C. B.; Stahlhut, S. G.; Li, M.; Gaspar, P.; Siedler, S.; Förster, J.; Maury, J.; Borodina, I.; Nielsen, A. T. Appl. Environ. Microbiol. 2015, 81, 4458-4476.

(9) Rodriguez, A.; Kildegaard, K. R.; Li, M.; Borodina, I.; Nielsen, J. Metab. Eng. 2015, 31, 181-188.

(10) Vannelli, T.; Wei Qi, W.; Sweigard, J.; Gatenby, A. A.; Sariaslani, F. S. Metab. Eng. 2007, 9, 142-151.

(11) Glavnik, V.; Simonovska, B.; Albreht, A.; Vovk, I. J. Planar Chromatogr. - Mod. TLC 2012, 25, 251-258.

(12) Jendresen, C. B.; Stahlhut, S. G.; Li, M.; Gaspar, P.; Siedler, S.; Förster, J.; Maury, J.; Borodina, I.; Nielsen, A. T. Appl. Environ. Microbiol. 2015, 81, 4458-4476.

(13) Dietrich, J. A.; McKee, A. E.; Keasling, J. D. Annu. Rev. Biochem. 2010, 79, 563-590.

(14) Li, B.; Ryan, P. W.; Ray, B. H.; Leister, K. J.; Sirimuthu, N. M. S.; Ryder, A. G. Biotechnol. Bioeng. 2010, 107, 290-301.

(15) Fan, M.; Andrade, G. F. S.; Brolo, A. G. Anal. Chim. Acta 2011, 693, 7-25.

(16) Nie, S. Science. 1997, 275, 1102-1106.

(17) Stiles, P. L.; Dieringer, J. A.; Shah, N. C.; Van Duyne, R. P. Annu. Rev. Anal. Chem. 2008, 1, 601-626.

(18) Cialla, D.; Pollok, S.; Steinbrücker, C.; Weber, K.; Popp, J. Nanophotonics 2014, 3, 383-411.

(19) Jarvis, R. M.; Brooker, A.; Goodacre, R. Faraday Discuss. 2006, 132, 281-292.

(20) Castillo, J. J.; Rindzevicius, T.; Rozo, C. E.; Boisen, A. Nanomater. Nanotechnol. 2015, 1-7.

(21) Yang, J.; Palla, M.; Bosco, F. G.; Rindzevicius, T.; Alstrøm, T. S.; Schmidt, M. S.; Boisen, A.; Ju, J.; Lin, Q. ACS Nano 2013 , 7, 5350-5359.

(22) Kim, A.; Barcelo, S. J.; Williams, R. S.; Li, Z. Anal. Chem. 2012, 84, 9303-9309.

(23) Peksa, V.; Jahn, M.; Štolcová, L.; Schulz, V.; Proška, J.; Procházka, M.; Weber, K.; Cialla-May, D.; Popp, J. Anal. Chem. 2015, 87, 2840-2844.

(24) Radu, A. I.; Ryabchykov, O.; Bocklitz, T. W.; Huebner, U.; Weber, K.; Cialla-May, D.; Popp, J. Analyst 2016, 141, 44474455 .

(25) Petryayeva, E.; Krull, U. J. Anal. Chim. Acta 2011, 706, 8-24.

(26) Stuart, D. A.; Yonzon, C. R.; Zhang, X.; Lyandres, O.; Shah, N. C.; Glucksberg, M. R.; Walsh, J. T.; Van Duyne, R. P. Anal. Chem. 2005, 77, 4013-4019.

(27) Morelli, L.; Bille Jendresen, C.; Zòr, K.; Rindzevicius, T.; Stenbæk Schmidt, M.; Toftgaard Nielsen, A.; Boisen, A. In Procedia Technology; In press, 2016.

(28) Sun, F.; Bai, T.; Zhang, L.; Liu, S.; Nowinski, A. K.; Jiang, S.; Yu, Q. Anal. Chem. 2014, 86, 2387-2394.

(29) Boca-Farcau, S.; Potara, M.; Simon, T.; Juhem, A.; Baldeck, P.; Astilean, S. Mol. Pharm. 2014, 11, 391-399.

(30) Jehlička, J.; Oren, A.; Vítek, P. Extremophiles 2012, 16, 507514.

(31) Giovannozzi, A. M.; Rolle, F.; Sega, M.; Abete, M. C.; Marchis, D.; Rossi, A. M. Food Chem. 2014, 159, 250-256.

(32) Lee, K.; Herrman, T. J.; Bisrat, Y.; Murray, S. C. J. Agric. Food Chem. 2014, 62, 4466-4474.

(33) Kim, A.; Barcelo, S. J.; Williams, R. S.; Li, Z. Anal. Chem. 2012, 84, 9303-9309.

(34) Stewart, S.; Fredericks, P. . Spectrochim. Acta Part A Mol. Biomol. Spectrosc. 1999, 55, 1641-1660.

(35) Garcia-Salas, P.; Morales-Soto, A.; Segura-Carretero, A.; Fernández-Gutiérrez, A. Molecules 2010, 15, 8813-8826.

(36) Naczk, M.; Shahidi, F. J. Chromatogr. A 2004, 1054, 95-111.

(37) Wierckx, N. J. P.; Ballerstedt, H.; Bont, J. a M. De; Wery, J. Appl. Environ. Microbiol. 2005, 71, 8221-8227.

(38) Krygier, K.; Sosulski, F.; Hogge, L. J. Agric. Food Chem. 1982 30, 330-334.

(39) Galanakis, C. M.; Goulas, V.; Tsakona, S.; Manganaris, G. A.;
Gekas, V. Int. J. Food Prop. 2013, 16, 382-396.

(40) Schmidt, M. S.; Hübner, J.; Boisen, A. Adv. Mater. 2012, 24 11-18.

(41) Wu, K.; Rindzevicius, T.; Schmidt, M. S.; Mogensen, K. B.; Hakonen, A.; Boisen, A. J. Phys. Chem. C 2015, 119, 20532062.

(42) Lauridsen, R. K.; Rindzevicius, T.; Molin, S.; Johansen, H. K. Berg, R. W.; Alstrøm, T. S.; Almdal, K.; Larsen, F.; Schmidt, M. S.; Boisen, A. Sens. Bio-Sensing Res. 2015, 5, 84-89.

(43) Miller, J. H. Experiments in Molecular Genetics; Cold Spring Harbor Laboratory, 1972.

(44) Rau, M. H.; Bojanovič, K.; Nielsen, A. T.; Long, K. S. BMC Genomics 2015, 16, 1051 .

(45) Krygier, K.; Sosulski, F.; Hogge, L. J. Agric. Food Chem. 1982, 30, 330-334.

(46) Schmidt, M. S.; Hübner, J.; Boisen, A. Adv. Mater. 2012, 24, $11-18$.

(47) Wu, K.; Rindzevicius, T.; Schmidt, M. S.; Mogensen, K. B Hakonen, A.; Boisen, A. J. Phys. Chem. C 2015, 119, 20532062.

(48) Clavijo, R. E.; Ross, D. J.; Aroca, R. F. J. Raman Spectrosc 2009, 40, 1984-1988.

(49) Mazet, V.; Carteret, C.; Brie, D.; Idier, J.; Humbert, B. Chemom. Intell. Lab. Syst. 2005, 76, 121-133.

(50) Pong, B.-K.; Lee, J. Y.; Trout, B. L. A Computational Study to Understand the Surface Reactivity of Gold Nanoparticles with Amines and DNA http://hdl.handle.net/1721.1/30380.

(51) Krueger, P. J. Spectrochim. Acta 1968, 24, 1055.

(52) Neelakantan, P. Proc. Indian Acad. Sci. - Sect. A 1963, 57, 330336.

(53) Analytical Applications of FT-IR to Molecular and Biological Systems; Durig, J. R., Ed.; Springer Netherlands: Dordrecht, 1980.

(54) Putschögl, M.; Zirak, P.; Penzkofer, A. Chem. Phys. 2008, 343, 107-120.

(55) Ferrer, C.; Lozano, A.; Agüera, A.; Girón, A. J.; FernándezAlba, A. R. J. Chromatogr. A 2011, 1218, 7634-7639. 


\section{For TOC only}

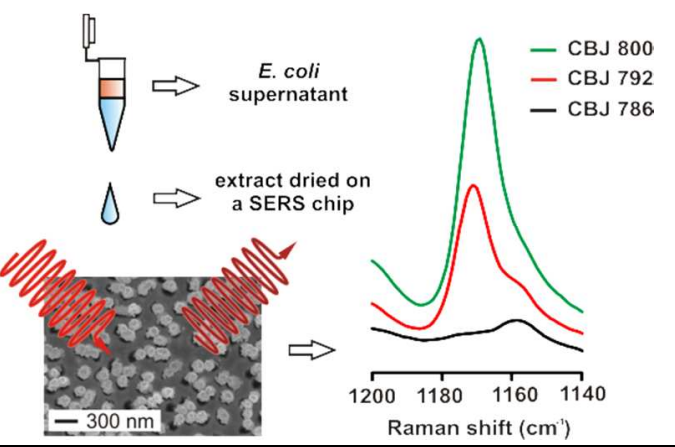

14

15

16

17

18

19

20

21

22

23

24

25

26

27

28

29

30

31

32

33

34

35

36

37

38

39

40

41

42

43

44

45

46

47

48

49

50

51

52

53

54

55

56

57

58

59

60 